\title{
How attentional boost interacts with reward: the effect of dopaminergic medications in Parkinson's disease
}

\author{
Szabolcs Kéri, ${ }^{1,2,3}$ Helga Nagy, ${ }^{4,5}$ Einat Levy-Gigi ${ }^{6}$ and Oguz Kelemen ${ }^{7}$ \\ ${ }^{1}$ Department of Physiology, Faculty of Medicine, University of Szeged, Szeged, Hungary \\ ${ }^{2}$ Gyula Nyírő Hospital, National Institute of Psychiatry and Addictions, Budapest, Hungary \\ ${ }^{3}$ Department of Cognitive Science, Budapest University of Technology and Economics, Budapest, Hungary \\ ${ }^{4}$ Department of Neurology, Semmelweis University, Budapest, Hungary \\ ${ }^{5}$ National Institute for Medical Rehabilitation, Budapest, Hungary \\ ${ }^{6}$ Department of Neurobiology and Ethology, The Brain and Behavior Laboratory, University of Haifa, Haifa, Israel \\ ${ }^{7}$ Bács-Kiskun County Hospital, Psychiatry Center, Kecskemét, Hungary
}

Keywords: attention, dopamine agonist, L-DOPA, memory, Parkinson's disease

\begin{abstract}
There is widespread evidence that dopamine is implicated in the regulation of reward and salience. However, it is less known how these processes interact with attention and recognition memory. To explore this question, we used the attentional boost test in patients with Parkinson's disease (PD) before and after the administration of dopaminergic medications. Participants performed a visual letter detection task (remembering rewarded target letters and ignoring distractor letters) while also viewing a series of photos of natural and urban scenes in the background of the letters. The aim of the game was to retrieve the target letter after each trial and to win as much virtual money as possible. The recognition of background scenes was not rewarded. We enrolled 26 drug-naïve, newly diagnosed patients with PD and 25 healthy controls who were evaluated at baseline and follow-up. Patients with PD received dopamine agonists (pramipexole, ropinirole, rotigotine) during the 12-week follow-up period. At baseline, we found intact attentional boost in patients with PD: they were able to recognize target-associated scenes similarly to controls. At follow-up, patients with PD outperformed controls for both target- and distractor-associated scenes, but not when scenes were presented without letters. The alerting, orienting and executive components of attention were intact in PD. Enhanced attentional boost was replicated in a smaller group of patients with PD $(n=15)$ receiving L-3,4-dihydroxyphenylalanine (L-DOPA). These results suggest that dopaminergic medications facilitate attentional boost for background information regardless of whether the central task (letter detection) is rewarded or not.
\end{abstract}

\section{Introduction}

There is a debate on the role of the midbrain dopaminergic centers in the regulation of motivation, salience processing and reward (Robbins \& Everitt, 2007; Berridge, 2007, 2012). A leading theory is that dopamine enhances reinforcement learning, resulting in the successful selection of rewarding actions during trial-and-error instrumental learning (Montague et al., 1996; Schultz et al., 1997; Samejima et al., 2005). Recent evidence suggests that reward may specifically modulate perception and memory. Seitz et al. (2009) presented visual orientation stimuli to thirsty individuals. Stimuli were paired with water administration as a reward. The authors demonstrated that visual learning was facilitated by stimulus-reward pairing without awareness of stimulus exposure and reward contingency (Seitz et al., 2009). Incidental learning elicited by reward signals may be linked to attentional modulation. When participants pair a target stimulus with reward, it may lead to attentional allocation

Correspondence: Dr S. Kéri, as above.

E-mails: keri.szabolcs.gyula@med.u-szeged.hu and szkeri2000@yahoo.com

Received 5 June 2013, revised 30 July 2013, accepted 2 August 2013 and better memory encoding not only for the target stimulus, but also on a non-relevant concurrently performed task (task-irrelevant perceptual learning; Seitz \& Watanabe, 2009).

Lin et al. (2010) designed a task in which central white letters were the targets to be remembered. Participants also viewed a series of photos of natural and urban scenes in the background of the letters. When there was no letter detection task, memory for scenes was at chance level. In contrast, when participants detected target letters, they also performed remarkably well on the recognition of background scenes. Distractor letters with another color that should be omitted did not encourage scene recognition (Fig. 1). The enhancement of background information (scenes) at behaviorally relevant points of time (i.e. when target letters are available) is also called the attentional boost effect (Swallow \& Jiang, 2010, 2011). A possible interpretation is that target letters elicited salient reward signals because the main aim of the task was their later recall. This signal may 'open' the attentional window leading to the incidental encoding of the background scene.

Ample evidence suggests that dopamine is implicated in attention regulation, and dopaminergic mechanisms may link salience/reward and attention (Nieoullon, 2002). For example, drugs enhancing 


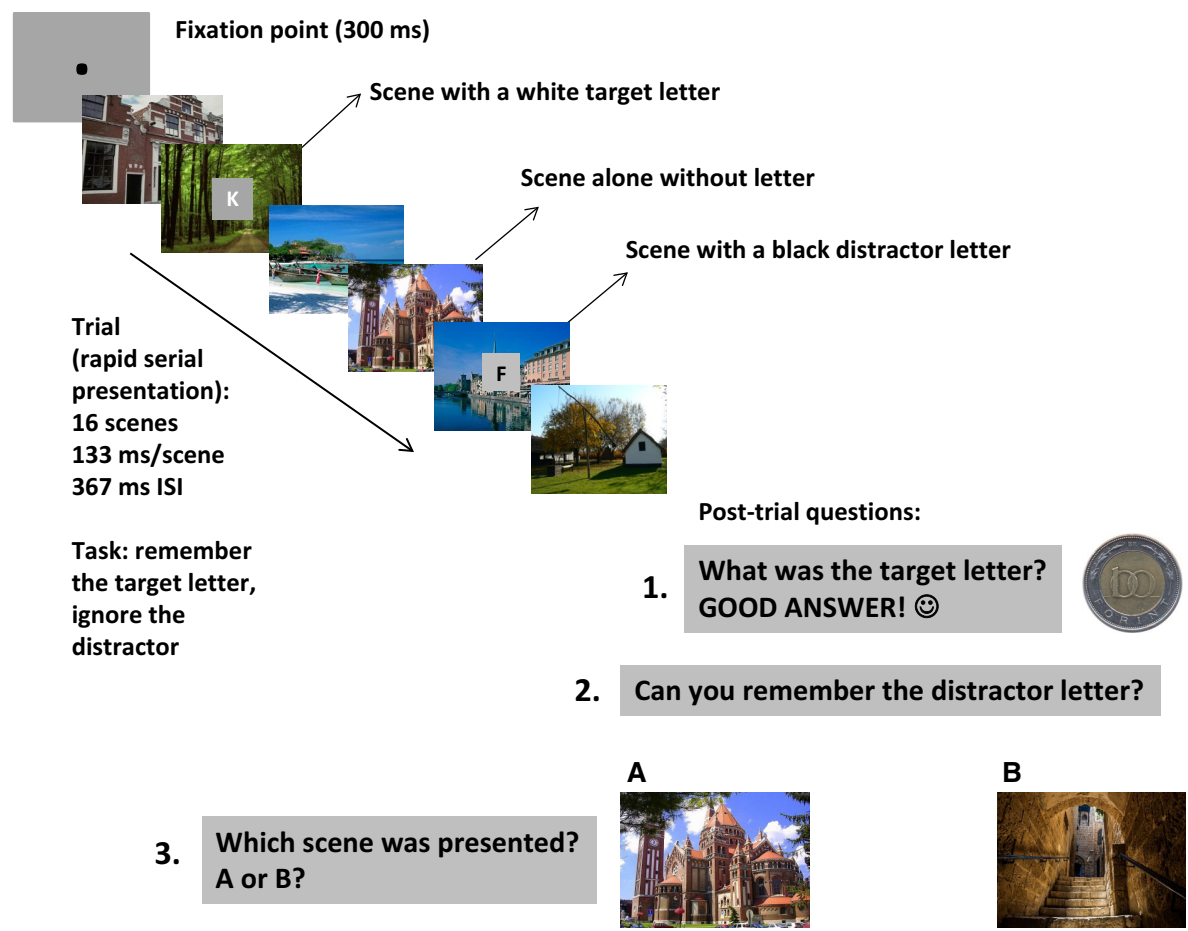

FIG. 1. Illustration of scene sequences. Two scenes appeared with white target letters that should be remembered, and two scenes appeared with black distractor letters that should be ignored. Following the trial, participants first typed the target letter, then the distractor letter and finally chose from the two test scenes. Only the recall of the target letter was rewarded. ISI, inter-stimulus interval.

dopaminergic transmission facilitate visual attention and memory via the modulation of the dorsal fronto-parietal attentional network (Müller et al., 2005; Tomasi et al., 2011), which is responsible for enhancing salient and attenuating irrelevant stimuli (Corbetta \& Shulman, 2002). Dopamine may play a vital role in the balanced and adaptive activation of functionally separated attentional networks of alerting, orienting and executive functions (Dang et al., 2012).

Parkinson's disease (PD) is characterized by the degeneration of dopaminergic neurons in the mesencephalon, resulting in various deficits in motor control, motivation and reinforcement learning, which is modulated by dopamine replacement therapy [dopamine precursor L-3,4-dihydroxyphenylalanine (L-DOPA) and dopamine receptor agonists; reviewed in Frank, 2005; Cools, 2006; Foerde \& Shohamy, 2011]. Some studies revealed attentional impairments in both early and advanced PD (e.g. Brown \& Marsden, 1988; Yamada et al., 1990; Hodgson et al., 1999; Muslimovic et al., 2005; Allcock et al., 2009; Zhou et al., 2012), whereas others did not do so (e.g. Rafal et al., 1984; Lee et al., 1999; Kingstone et al., 2002; Cristinzio et al., 2012). Dopaminergic signals in the striatum and its interaction with the prefrontal cortex would be especially critical in the regulation and integration of higher-level processes, such as attention and cognitive control (Cools, 2011).

The first aim of the present study was to examine how dopamine participates in the regulation of attentional boost by the investigation of patients with PD before and after the administration of dopaminergic medications. We hypothesized that patients with $\mathrm{PD}$ receiving dopamine agonists would improve scene recognition performance when scenes are presented with rewarded target letters. Second, we studied the relationship between attentional boost and traditional components of attention (alerting, orienting, executive). Third, we explored the relationship between changes in clinical symptom and psychological trait (motor symptoms, depression, impulsivity) and attentional boost before and after dopamine agonist therapy. Finally, we assessed a separate group of patients with PD receiving L-DOPA medication to test the reproducibility of the results and to examine whether the observed effects are specific for dopamine agonists or not.

\section{Materials and methods}

\section{Participants}

In the first sample, we recruited 26 newly diagnosed, drug-naive patients with PD and 25 control individuals (acquaintances of hospital staff and non-biological family members of patients matched for age, gender, education and IQ; Table 1). After baseline testing in an unmedicated state, patients received dopamine agonist therapy and were followed-up for 12 weeks [pramipexole: $n=10$, mean dose at follow-up: $4.5 \mathrm{mg} / \mathrm{day}$, range $3.0-6.5 \mathrm{mg} / \mathrm{day}$; ropinirole: $n=10$, mean dose at follow-up: $6.0 \mathrm{mg} /$ day, range: $2.5-7.5 \mathrm{mg} /$ day; rotigotine: $n=6 ; 6 \mathrm{mg} / 24 \mathrm{~h}$; levodopa equivalent dose (LED): $250 \mathrm{mg} /$ day; Tomlinson et al., 2010]. After the 12-week follow-up period, participants were re-evaluated. In the second sample, we included 15 patients with recent-onset PD receiving L-DOPA monotherapy and 15 matched healthy controls (Table 2). We assessed the second sample only once. The diagnosis of PD was based on the UK Parkinson's Disease Society Brain Bank Clinical Diagnostic Criteria (Hughes et al., 1992). All participants gave written informed consent prior to their participation. All procedures were approved by the Human Investigation Review Board (protocol number: 2697/2011) in accordance with the declaration of Helsinki (1964).

\section{Clinical and demographic assessment}

Trained neurologists and clinical psychologists, who were blind to diagnosis, attention test performance and medication status, administered the scales and interviews. The severity of PD 
TABLE 1. Clinical and demographic characteristics of the participants in the follow-up study

\begin{tabular}{|c|c|c|}
\hline & Controls & Parkinson's disease (PD) \\
\hline $\begin{array}{l}\text { Number of participants } \\
\text { (male/female) }\end{array}$ & $25(18 / 7)$ & $26(17 / 9)$ \\
\hline Age (years) & $48.6(6.5)$ & $49.2(7.4)$ \\
\hline Education (years) & $13.9(7.4)$ & $14.1(6.6)$ \\
\hline Full-scale IQ (WAIS-R) & $105.4(10.1)$ & $104.6(11.0)$ \\
\hline $\begin{array}{l}\text { Socioeconomic status } \\
\text { (Hollingshead) }\end{array}$ & $35.6(13.9)$ & $35.5(17.3)$ \\
\hline $\begin{array}{l}\text { Time since onset of first } \\
\text { symptoms (months) }\end{array}$ & - & $15.4(7.4)$ \\
\hline $\begin{array}{l}\text { No. of patients in } \\
\text { Hoehn-Yahr Stage }\end{array}$ & - & $\begin{aligned} 1.0 & : 4 \\
1.5 & : 13 \\
2 & : 9\end{aligned}$ \\
\hline \multicolumn{3}{|l|}{ UPDRS total } \\
\hline Baseline & - & $33.5(5.2)$ \\
\hline $\begin{array}{l}\text { Follow-up } \\
\text { UPDRS IU (motor) }\end{array}$ & - & $30.2(4.6)^{*}$ \\
\hline Baseline & - & $25.1(5.0)$ \\
\hline Follow-up & - & $21.6(5.4)^{\dagger}$ \\
\hline \multicolumn{3}{|l|}{ HAM-D } \\
\hline Baseline & $3.5(2.5)$ & $5.7(4.2)$ \\
\hline Follow-up & $3.8(2.3)$ & $2.6(2.2)^{*}$ \\
\hline \multicolumn{3}{|l|}{ BIS-11 } \\
\hline \multicolumn{3}{|l|}{ Total score } \\
\hline Baseline & $62.4(9.8)$ & $60.7(10.9)$ \\
\hline Follow-up & $62.5(9.5)$ & $64.1(12.0)^{\S}$ \\
\hline \multicolumn{3}{|l|}{ Attentional score } \\
\hline Baseline & $16.7(3.9)$ & $15.5(3.4)$ \\
\hline Follow-up & $16.9(3.8)$ & $17.0(3.3)^{\pi}$ \\
\hline
\end{tabular}

Data are mean (standard deviation). BIS-11, Barrett Impulsiveness Scale-11; HAM-D, Hamilton Depression Scale; UPDRS, Unified Parkinson's Disease Rating Scale; WAIS-R, Wechsler Adult Intelligence Scale, revised. The two groups did not differ in gender distribution, age, education, IQ, and socioeconomic status (chi-square and $t$-test, $P>0.5$ ). ${ }^{*} t_{50}=2.50, P=0.02$ (baseline vs. follow-up). ${ }^{\dagger} t_{50}=2.39, P=0.02$ (baseline vs. follow-up). ${ }^{*} \mathrm{PD}$ [baseline] $>$ control[baseline]; PD $\mathrm{Pbaseline}]>\mathrm{PD}$ [follow-up]. ${ }^{\S} \mathrm{PD}$ [follow-up] $>\mathrm{PD}$ [baseline]. ${ }^{\top} \mathrm{PD}[$ follow-up] $>\mathrm{PD}$ [baseline](all analyses in c-f: ANOva followed by Tukey HSD, $P<0.05$ ).

symptoms was evaluated using the Hoehn-Yahr Scale (Hoehn \& Yahr, 1967) and the Unified Parkinson's Disease Rating Scale (UPDRS; Lang \& Fahn, 1989). For the diagnosis of possible mental disorders, we used the Structured Clinical Interview for DSM-IV Axis I Disorders, Clinician Version (First et al., 1996). Depressive symptoms, impulse control disorders and pathological gambling were screened with the Hamilton Depression Rating Scale (HAM-D; Hamilton, 1960), Minnesota Impulsive Disorders Interview (MIDI; Christenson et al., 1994) and South Oaks Gambling Screen (SOGS; Lesieur \& Blume, 1987), respectively.

We also administered the Barratt Impulsiveness Scale-11 (BIS-11) evaluating three dimensions of impulsivity (motor impulsivity, attentional impulsivity and non-planning; Patton et al., 1995). Beyond the total BIS-11 score, we focused on attentional impulsivity because this dimension is the most definitive measure of impulsivity in PD (Antonini et al., 2011), and this dimension of the BIS-11 is the most relevant in relation to attentional functions.

Socioeconomic status was described with the Hollingshead FourFactor Index (Hollingshead, 1975), and general cognitive functions were assessed with the revised version of the Wechsler Adult Intelligence Scale (Wechsler, 1981).

\section{Attentional boost test (ABT)}

Stimuli were presented on a VP2765-LED-27" monitor (ViewSonic, Walnut, CA, USA; refresh rate: $60 \mathrm{~Hz}$, resolution: $1920 \times$
TABLE 2. Clinical and demographic characteristics of the participants in the replication study

\begin{tabular}{lcc}
\hline & Controls & Parkinson's disease (PD) \\
\hline $\begin{array}{l}\text { Number of participants } \\
\text { (male/female) }\end{array}$ & $15(10 / 5)$ & $15(9 / 6)$ \\
Age (years) & $59.4(7.0)$ & $60.4(4.1)$ \\
Education (years) & $14.6(4.4)$ & $14.2(4.9)$ \\
Full-scale IQ (WAIS-R) & $110.6(10.8)$ & $109.4(12.3)$ \\
Socioeconomic status & $39.4(12.0)$ & $40.1(13.8)$ \\
(Hollingshead) & & $30.6(8.5)$ \\
Time since onset of first & - & $1.0: 1$ \\
symptoms (months) & & $1.5: 5$ \\
No. of patients in & - & $2: 9$ \\
Hoehn-Yahr Stage & & $690.0(365.7)$ \\
& & $36.7(6.9)$ \\
L-DOPA (mg/day) & & $7.3(3.8)^{*}$ \\
UPDRS total & & $62.1(7.9)$ \\
UPDRS III (motor) & & $16.9(3.9)$ \\
HAM-D & $3.5(2.3)$ & \\
BIS-11 & & \\
Total score & $60.3(9.6)$ &
\end{tabular}

Data are mean (standard deviation) with the exception of gender. BIS-11, Barrett Impulsiveness Scale -11; HAM-D, Hamilton Depression Scale; UPDRS, Unified Parkinson's Disease Rating Scale; WAIS-R, Wechsler Adult Intelligence Scale, revised. The two groups did not differ in gender distribution, age, education, IQ, and socioeconomic status (chi-square and $t$-test, $P>0.5)$. ${ }^{*} t_{28}=-3.32, P=0.003$.

1080 pixel; viewing distance: $50 \mathrm{~cm}$; output luminance: $65 \mathrm{~cd} / \mathrm{m}^{2}$ ) controlled by a personal computer (Dell XPS workstation). We used photographs of natural and urban scenes (size: $28^{\circ}$ of visual angle), as described previously (Levy-Gigi \& Kéri, 2012; Szamosi et al., 2013).

The experimental trials included rapid serial presentations of scenes (exposure time: $133 \mathrm{~ms} / \mathrm{scene}$; inter-stimulus interval: $367 \mathrm{~ms}$ ). Participants were presented with 16 scenes. Four of the 16 scenes contained superimposed letters in their center (two white target letters; two black distractor letters; Fig. 1). Twelve scenes in the sequence contained no letters. The task was to remember the target letters. Participants were explicitly instructed to ignore and forget the distractor letters. Following each trial (presentation of 16 scenes: two scenes with target; two scenes with distractor; and 12 scenes alone in a pseudorandom order), participants were first asked to type the target letter, which was followed by immediate feedback ('Good answer!' with a smiling cartoon face and a symbolic monetary reward of 100 Hungarian Forints; wrong answers were not followed by feedback). Following the response, we asked the participants to type the distractor letter if they remembered that. Immediately after the letter recall task, two scenes ('A' and 'B') were exposed for $3000 \mathrm{~ms}$. One of these scenes was from the sequence (serial position: 6-14). The other scene was not presented in the sequence. The task was to decide which of the two scenes appeared in the sequence by pressing one of two keys ('A' or 'B'; Fig. 1). The scene presented in this recognition phase could be a scene without a letter, with a target letter, or with a distractor letter in the sequence. In task introduction and instructions, it was emphasized that the main aim of the game was to remember the target letter, which led to reward. Recall of distractor letters and scene recognition were not followed by feedback. There were 300 intermixed trials $(10$ blocks of 30 trials) separated by breaks. Before the test, participants received a training session (30 trials). However, they did not see the test scenes before the rapid serial presentation trials. The dependent 
measures were the percentage of correctly recalled letters and the percentage of correctly recognized scenes.

The task described above was different from the original procedure used by Lin et al. (2010): (i) correct responses in the letter recall phase were rewarded; (ii) two scenes had white (target) and two scenes black (distractor) letters during the 16-item serial visual presentation stream; (iii) participants completed a recall task for both target and distractor letters. However, participants were asked to ignore, suppress and not remember the distractors, which is similar to directed forgetting paradigms (Baddeley et al., 2009).

\section{Attention network test (ANT)}

The method has been extensively documented in previous studies (Fan et al., 2002, 2005, 2009). The ANT has been used in many studies on the genetics, development and clinical disorders of attention (e.g. Posner, 2008). The test-retest reliability of the ANT was adequate in healthy individuals and patients with schizophrenia (Hahn et al., 2011). We used this procedure in the present study. The apparatus for stimulus presentation and response collection was the same as in the ABT.

The experimental trials consisted of the following parts: (i) first fixation (duration: 400-1600 ms); (ii) cue presentation (duration: $100 \mathrm{~ms}$ ); (iii) second fixation (duration: $400 \mathrm{~ms}$ ); (iv) target presentation (maximum duration: $1700 \mathrm{~ms}$ ). The target stimulus consisted of five horizontal arrows or lines presented above or below the fixation cross. We asked the participants to indicate the direction of the central arrow by pressing keys representing left or right direction on the computer keyboard. Flankers next to the central arrow were lines (neutral target condition) or arrows with the same (compatible) or opposite (incompatible/conflict) direction. The cue stimuli could be a spatial cue (presented above or below the fixation cross indicating the location of the target), a double cue (presented above and below the center) and a center cue (presented in the center). There were trials with no cues.

First, participants received 24 training trials with feedback. Second, we presented 288 trials $(4$ cues $\times 3$ targets $\times 8$ repetitions per block $\times 3$ blocks). The sequence of trials was pseudo-randomized. There was no feedback. The dependent measures were response time and error rate differences reflecting the alerting, orienting and executive components of attention. These indexes were calculated as follows: alerting-no cue minus double cue; orienting-previous center cue minus previous spatial cue; executive (conflict)-incompatible targets minus targets.

\section{Statistical analysis}

We used statistica 11 (StatSoft, Tulsa, USA) and Prism 6 (GraphPad, La Jolla, USA) software for data analysis. First, we ran goodness-of-fit analysis (Kolmogorov-Smirnov test for normality of data distribution). We used repeated measures analyses of variance (ANOvas), followed by Tukey Honestly Significant Difference (HSD) post hoc tests. In the analysis of the letter recall part of the ABT, the between-subjects factor was group (PD vs. controls) and the withinsubjects factors were time (baseline vs. follow-up) and stimulus type (target vs. distractor letters). In the analysis of the scene recognition part of the ABT, the between-subjects factor was group (PD vs. controls), and the within-subjects factors were time (baseline vs. follow-up) and stimulus type (scenes associated with targets, distractors and scenes alone). In the ANT, we ran separate ANOvAs for mean response time and error rate, response time indexes, and error rate indexes with the group as the between-subjects factor and time as the within-subjects factor. The same ANOvA design was used for the analysis of rating scales (HAM-D and BIS-11). In the replication sample, time was not a within-subjects factor because it was a cross-sectional study. To explore the relationship between changes in ABT and rating scales, we calculated Pearson's product moment correlation coefficients, corrected for multiple comparisons with the Bonferroni method. Demographic parameters were compared with two-tailed $t$-tests and chi-square tests. The level of statistical significance was $\alpha<0.05$.

\section{Results}

\section{General description of the sample from the follow-up study}

Table 1 depicts the clinical and demographic characteristics of the patients with PD and control individuals. One patient with PD had specific phobia. None of the other patients and controls exhibited DSM-IV Axis I disorders at baseline and follow-up. MIDI/SOGS revealed no impulse control disorders at baseline and follow-up. Patients with PD and control individuals did not differ in age, gender, education, IQ and socioeconomic status. Patients with PD scored higher than controls on the HAM-D scale at baseline but not at follow-up. Patients with PD and controls did not differ significantly in BIS-11 scores, although patients with PD achieved higher scores at follow-up relative to baseline (Table 1).

\section{Detection of target and distractor letters}

Patients with PD and control volunteers displayed similar letter detection performances at baseline and follow-up (ANOvA, $P>0.5$ ). As expected, letter detection performance for targets was higher than that for distractors $(P<0.0001$; Fig. 2).

\section{ABT}

Figure 3 depicts the results from the scene recognition test. The ANOvA revealed significant main effects of group $\left(F_{1,49}=7.0, P<0.05\right.$, $\left.\eta^{2}=0.13\right)$, time $\left(F_{1,49}=40.06, P<0.0001, \eta^{2}=0.45\right)$ and stimulus type $\left(F_{2,98}=233.66, P<0.0001, \eta^{2}=0.83\right)$. There were significant two-way interactions between group and time $\left(F_{1,49}=33.50, P<\right.$ $\left.0.0001, \eta^{2}=0.41\right)$, group and stimulus type $\left(F_{2,98}=3.55, P<0.05\right.$, $\left.\eta^{2}=0.07\right)$, and time and stimulus type $\left(F_{2,98}=6.74, P<0.005\right.$, $\left.\eta^{2}=0.12\right)$. We also found a three-way interaction among group, time and stimulus type $\left(F_{2,98}=7.75, P<0.005, \eta^{2}=0.14\right)$. A control analysis indicated no significant differences among patients receiving different dopamine agonists $(F<1, P>0.5)$.

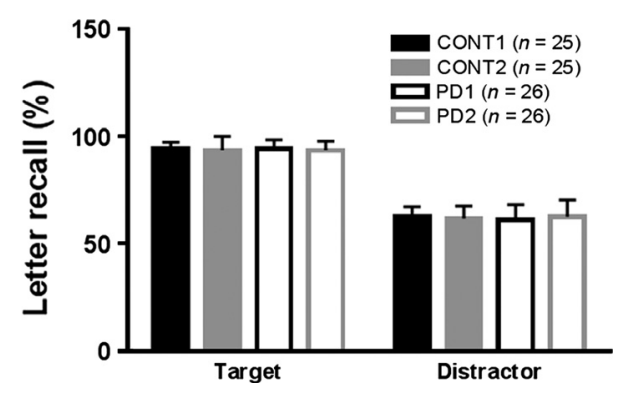

FIG. 2. Letter recall performance in Parkinson's disease (PD) and healthy control individuals (CONT) at baseline (1) and follow-up (2). There was no significant difference between the two groups. Error bars indicate SDs. 


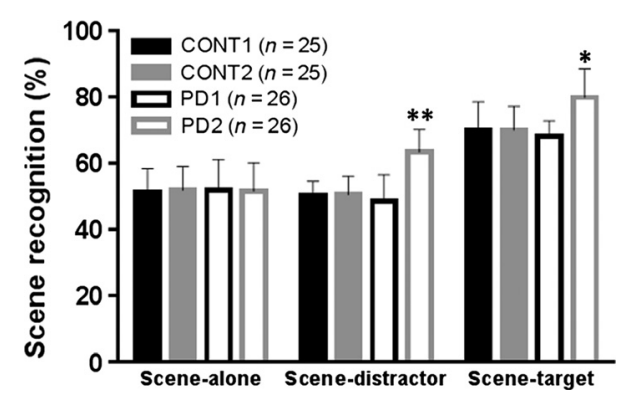

FIG. 3. Recognition performance for scenes presented alone, with distractor letters, and with target letters in Parkinson's disease (PD) and healthy control individuals (CONT) at baseline (1) and follow-up (2). At follow-up, patients with PD receiving dopamine agonists outperformed control individuals for scenes with targets $\left({ }^{*} P<0.05\right.$, Tukey HSD test) and scenes with distractors $(* * P<0.001$, Tukey HSD test). Error bars indicate SDs.

Tukey HSD tests yielded no difference between patients with PD and control individuals at baseline $(P>0.5)$. At follow-up, patients with PD showed higher levels of scene recognition performance relative to control individuals when distractors and targets were presented with the scenes in the trial sequence $(P<0.001$ and $P<0.05$, respectively). Within-group comparisons revealed good test-retest characteristics in control individuals (baseline vs. followup: $P>0.5$; correlations: $r>0.7$ ). In PD, we observed enhanced scene recognition performances at follow-up relative to baseline when scenes were presented with targets and distractors $(P<0.01)$, but not when scenes were presented alone in the trial sequence $(P>0.5$; Fig. 3). There was a significant positive relationship between recognition improvements for scenes presented with targets and distractors in the trial sequence $(r=0.72, P<0.001)$. In patients with $\mathrm{PD}$, there was no significant correlation between the recall of distractor letters and the recognition of scenes paired with distractors $(r=0.16)$.

\section{ANT}

The ANOVA conducted on the mean response time indicated significant main effects of group $\left(F_{1,49}=14.73, P<0.001, \eta^{2}=0.23\right)$ and time $\left(F_{1,49}=10.37, P<0.005, \eta^{2}=0.17\right)$. The interaction between group and time was also significant $\left(F_{1,49}=7.53\right.$, $\left.P<0.05, \eta^{2}=0.13\right)$. The post hoc analysis confirmed that patients with PD responded slower than controls at baseline $(P<0.0001)$ and follow-up $(P<0.05)$. Within-group comparisons revealed that in $\mathrm{PD}$ the response time was faster at follow-up relative to baseline $(P<0.005)$, whereas in control volunteers response latency showed a marked stability over time (baseline vs. follow-up, $P=0.98$ ). Other measures of the ANT did not show significant alterations in PD compared with control individuals $(P>0.1$; Figs 4 and 5$)$. There were no significant correlations between ANT and ABT measures $(-0.2<r<0.2, P>0.1)$.

\section{Correlation between changes in clinical scales and $A B T$}

We calculated correlation coefficients between changes in UPDRS, HAM-D and BIS-11 attention scores and changes in scene recognition when scenes were presented with targets and distractors (change: follow-up-baseline). Given that this analysis was exploratory, we used Bonferroni corrections for multiple comparisons. We found significant correlation between changes in BIS-11 attention scores and changes in recognition performance for distractor-associated scenes $(r=0.66$,

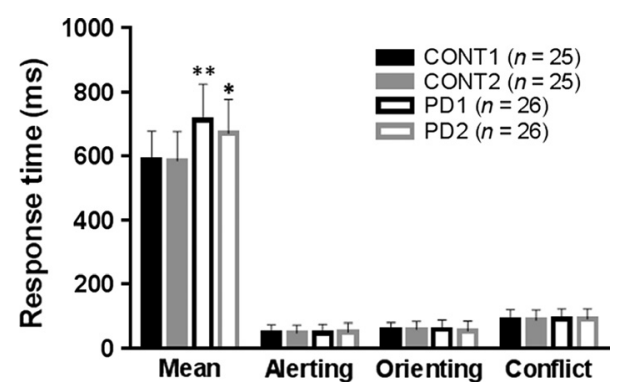

FIG. 4. Mean response time and index values from the attention network test (ANT). Patients with Parkinson's disease (PD) responded slower than control individuals (CONT) at baseline $(1)(* * P<0.0001$, Tukey HSD test) and follow-up (2) $(* P<0.05$, Tukey HSD test). There were no significant differences in index values (alerting, orienting, conflict). Error bars indicate SDs.

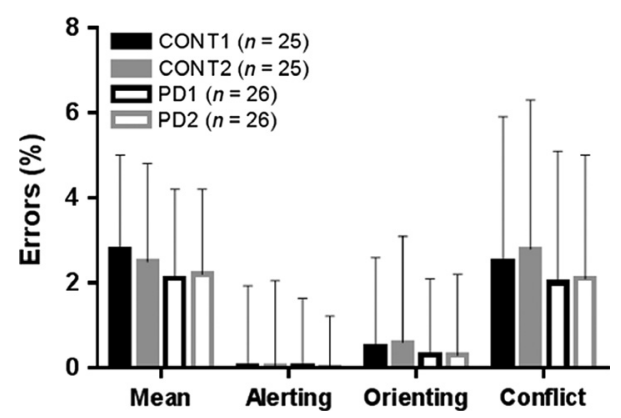

FIG. 5. Mean error rates (\%) from the attention network test (ANT). There were no statistically significant differences between patients with Parkinson's disease (PD) and control individuals (CONT). Error bars indicate SDs.

$P<0.001$; other correlations: $-0.4<r<0.4, P>0.05)$. LED did not correlate with BIS-11 and attentional boost $(-0.3<r<0.3$, $P>0.1)$.

\section{Replication in patients with $P D$ receiving $L-D O P A$}

Table 2 summarizes the characteristics of the replication sample. Patients with PD and controls were matched for demographic parameters. Two patients with PD had DSM-IV major depressive disorder, and one patient had generalized anxiety disorder. No impulse controls disorders were diagnosed. Patients with PD displayed higher scores than control individuals on HAM-D (Table 2).

Patients with PD and control individuals performed similarly on the letter detection task [patients with PD-target: 93.2\% (SD = 3.2), distractor: $61.3 \%(\mathrm{SD}=4.6)$; controls-target: $93.3 \% \quad(\mathrm{SD}=3.1)$, distractor: $61.6 \%(\mathrm{SD}=5.6) ; P>0.5]$.

The ANOVA conducted on the scene recognition performance revealed significant main effects of group $\left(F_{1,28}=35.73, P<0.0001\right.$, $\left.\eta^{2}=0.56\right)$ and stimulus type $\left(F_{2,56}=63.16, P<0.0001, \eta^{2}=0.69\right)$. The two-way interaction between group and stimulus type was significant $\left(F_{2,56}=4.93, P<0.05, \eta^{2}=0.15\right)$. Tukey HSD tests indicated that patients with PD showed higher levels of scene recognition than control individuals when scenes were presented with targets and distractors in the trial sequence $(P<0.01$; Fig. 6$)$.

We calculated correlations between scene recognition, HAM-D, UPDRS and BIS-11 attention score. In the whole sample $(n=30)$, we found a significant positive correlation between BIS-11 attention 


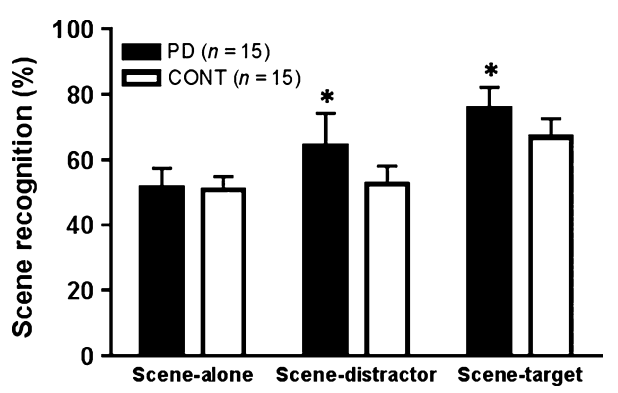

FIG. 6. Recognition performance for scenes presented alone, with distractor letters, and with target letters in Parkinson's disease (PD) receiving L-3, 4-dihydroxyphenylalanine (L-DOPA) and healthy control individuals (CONT). Patients with PD outperformed controls for scenes with targets and distractors $(* P<0.01$, Tukey HSD test).

score and recognition performance for distractor-associated scenes $(r=0.41, P=0.02)$.

\section{Discussion}

\section{Summary of the results}

We observed no evidence for attentional dysfunctions in drug-naïve, young patients with PD. However, at follow-up when patients with PD received dopamine agonists, we found enhanced attentional boost for both target- and distractor-associated scenes: patients with PD recognized scenes better than control individuals did when scenes were presented with either targets or distractors in the encoding phase. Higher impulsive attention was associated with better scene recognition performance when scenes were presented with distractors in the encoding phase. This finding is against the hypothesis that dopamine selectively enhances memory for reward/targetassociated background information. Instead, dopamine enhances attentional impulsivity and facilitates memory for information presented with both targets and distractors. However, there was a specific association between attentional impulsivity and distractorassociated recognition performance. Dopamine agonists and L-DOPA had no general enhancing effect on memory because recognition memory for scenes presented alone was not encouraged.

Enhanced attentional boost was not related to the alerting, orienting or executive components of attention, which were not affected by dopaminergic medications. We replicated enhanced attentional boost in elderly patients with PD who received L-DOPA. Despite the differences in patient characteristics and design, the results were consistent in the two PD groups.

\section{Mechanisms of attentional boost}

Swallow \& Jiang (2011) delineated several hypotheses to explain the attentional boost effect. The first is attentional cueing, which is based on an orienting response to the target leading to a concurrently enhanced processing of the background scene. Attentional cueing may occur concurrently with perceptual learning when target and scene are assembled into a single object (Driver \& Baylis, 1989). Based on our findings, however, a simple attentional cueing does not sufficiently explain attentional boost because we did not find any connection with alerting and orienting of visual attention. In a similar paradigm to that used in the present study, Leclercq \& Seitz (2012) found poor memorization for scenes that were preceded by an auditory alerting cue. However, for target-paired scenes, memory was enhanced when an alerting cue preceded the target, but only when the cue was available only on a subset of trials (Leclercq \& Seitz, 2012).

Another possibility is that the target elicits a reward/salience signal because the proper identification and recall of the target letter was the main purpose of the task, and therefore it was indirectly reinforced in the experimental conditions (Seitz \& Watanabe, 2009; Swallow \& Jiang, 2011; but see also Tosoni et al., 2013). In the present study, we used a direct reward. This reward might 'widen the window of attention' facilitating the encoding of the background scene. The hippocampal formation may play a pivotal role in this encoding process because individuals with hippocampal atrophy had weak attentional boost (Szamosi et al., 2013). Shohamy \& Wagner (2008) showed that the interaction between midbrain dopaminergic centers (ventral tegmental area/substantia nigra) and the hippocampal formation is essential for associative encoding (see also Wimmer et al., 2012). There is evidence that in the hippocampus dopaminergic modulation of attention is important in the selection of relevant and salient information (Muzzio et al., 2009). We propose that similar mechanisms may be implicated in attentional boost, which is intact in early-stage PD when dopaminergic loss is not pronounced in the midbrain-hippocampal system (Foerde et al., 2013). If dopaminergic medications are used in this stage of the disease, patients will demonstrate enhanced attentional boost outperforming healthy unmedicated individuals.

An intriguing finding was that patients with PD receiving dopaminergic medications displayed enhanced attentional boost not only in the case of rewarded targets, but also in the case of distractors. In other words, they might encode scenes not only at behaviorally rewarded points of time, but also at behaviorally inhibited occasions when central stimuli (distractors) had to be ignored. In contrast, at behaviorally neutral points (scenes alone), there were no such effects. Moreover, patients with PD successfully ignored the distractors, and yet they had increased levels of recognition for scenes exposed concurrently with the omitted distractors, suggesting that they failed to 'close the window of attention' spreading to the background scene. In a different paradigm, Cools et al. (2010) also revealed that dopaminergic medications decreased 'distractor resistance' in PD (see also Moustafa et al., 2008).

\section{Dopamine, attention and $P D$}

The results of the present study are consistent with the findings of previous reports that found no severe attentional dysfunction in early-stage PD (e.g. Rafal et al., 1984; Della Sala et al., 1986; Cossa et al., 1989; Lee et al., 1999; Kingstone et al., 2002; Koerts et al., 2009; Cristinzio et al., 2012), and indicate that dopamine agonists do not affect alerting, orienting and executive attention. Other researchers suggested that attentional dysfunction in PD is confined to internal cognitive control mechanisms (Brown \& Marsden, 1988; Bennett et al., 1995). However, using the ANT, Zhou et al. (2012) demonstrated a selective deficit of the orienting network, although results also revealed that alerting and executive components might be compromised in a more advanced stage of the disease (see also Allcock et al., 2009; Vandenbossche et al., 2012).

Results from animal models and human pharmacological studies suggest that dopamine is specifically related to the executive attentional network (Marrocco \& Davidson, 1998). However, Robbins (2002) argued that in animals the systematic administration of dopaminergic agents predominantly affects response latency, premature responses and omissions via the dorsal and ventral striatal systems. The administration of dopamine agonists in 
humans also modulates striatal and midbrain responses to reward (Riba et al., 2008; Abler et al., 2009). Our findings are consistent with the response speed hypothesis of systematic dopaminergic effects (Robbins, 2002) because the sole change after the administration of dopamine agonists was shorter mean reaction times. Dopamine agonists had no noticeable effects on the altering, orienting and executive measures in contrast to attentional boost, which was significantly enhanced. This suggests that the attention indexes, as measured by the ANT, are dissociable from attentional boost.

\section{Atypical attentional boost and impulsivity: relevance for translational neuroscience}

What is the practical relevance of enhanced attentional boost? We found that changes in BIS-11 attentional impulsivity correlated with atypical attentional boost (enhanced memory for distractor-associated scenes). Housden et al. (2010) also reported impulsivity in medicated patients with PD. In the ABT, target stimuli are salient and rewarded, leading to the enhanced encoding of the background scene. Distractors are not rewarded, and therefore there is no enhanced encoding of the background scene. This latter omission of distractor-associated scenes is disinhibited in patients with PD receiving dopaminergic medications, which is in accordance with our previous results from a simple associative learning task (Nagy et al., 2012).

The widespread use of dopamine receptor agonists in PD drew attention to the adverse psychological effects of these medications, with a special reference to impulse control disorders (pathological gambling, hypersexuality, compulsive buying and eating). In dopamine dysregulation syndrome, which is typically observed when short-acting and high-potency dopaminergic medications are used, patients exhibit addictive drug seeking and consumption, elevated mood, dyskinesias, and withdrawal symptoms (dysphoria and anxiety in response to dose reduction; Weintraub \& Nirenberg, 2013). Researchers postulated that these adverse effects are related to the mesencephalic-ventral striatal dopaminergic pathways, which are essential in reward, motivation and mood regulation; specifically, dopamine agonists may disrupt risk evaluation in the striatum in patients with impulse control disorders (Lawrence et al., 2003; Rao et al., 2010; Voon et al., 2011). This is also consistent with the animal studies reviewed above (Robbins, 2002).

In our study, none of the patients developed impulsive-compulsive behavior, although we observed a significant elevation of BIS-11 scores after dopaminergic medications relative to the unmedicated baseline. This is consistent with the findings of Isaias et al. (2008) who reported increased impulsivity in medicated patients with PD. Antonini et al. (2011) showed a trend toward higher BIS11 attention impulsivity even in unmedicated patients with PD who displayed clinically meaningful impulse control disorders before the administration of dopaminergic medications. Canesi et al. (2012) found no significant difference in items of BIS-11 amongst groups of patients with PD and control individuals, although BIS-11 score positively correlated with LED and was numerically higher in medicated patients with PD relative to control individuals. In the present study, this correlation was not significant, possibly because of the small sample size and narrow range of doses.

\section{Limitations and future directions}

First, the sample size was too small to conduct powerful correlation analyses taking into account all confounding and moderator variables. Second, it is still unclear whether subtle changes in impulsivity are sufficient to predict subsequent development of impulse control disorders and dopamine dysregulation syndrome. We did not assess patients with the above-mentioned clinical symptoms, but high BIS-11 scores may be indicative for vulnerability to impulse control disorders (Antonini et al., 2011).

The third potential limitation stems from the task design. Simultaneous instructions to ignore and then report the distractors may be confusing, and makes them relevant and salient. Despite the fact that the distractors were not rewarded, it is likely that they were selected by contingent capture of attention (Folk et al., 1992), and then intentionally forgotten or not reported. However, contingent capture of attention facilitated scene memory only when patients with PD received dopaminergic therapy, which, on the other hand, did not affect intentional forgetting of distractors.

Our task was similar to directed forgetting designs (Baddeley et al., 2009) when memory for 'to-be-forgotten' items is weaker, but regularly above chance level. The above-chance level of performance for distractor letters suggests reliable responses (i.e. extreme below-chance performance might suggest that participants intentionally did not report distractor letters that they remembered). One may assume that participants simply knew that the distractor will be asked and thus attempted to remember it better and with it they encoded the scenes. However, our results do not indicate that participants attempted to remember the distractor-associated scenes better because scene recognition performance was at the chance level in this condition, which was similar to the case when scenes were presented alone. Moreover, in the dopamine replacement condition, a boosting effect was observed for the recognition of distractor-associated scenes but not for the recall of distractor letters, which indicates an intriguing dissociation between the recall of the central stimulus (letters) and the recognition of the background information (scenes). A possible explanation may be that the short-term memory systems responsible for maintaining the letters and the neural systems responsible for the attentional boost are not equally affected by PD and dopaminergic medications, and that medicated patients with PD have less control over distracting items (e.g. Moustafa et al., 2008).

The neuronal correlates of attentional boost and its pharmacological modulation need to be investigated using functional neuroimaging methods. Swallow et al. (2012) provided evidence that responding to target stimuli at behaviorally relevant points of time enhanced activity in early visual cortical areas, but it is not clear how it affects memory for contextual background images. Although our current results and data from individuals with hippocampal atrophy (Szamosi et al., 2013) suggest the relevance of midbrain dopaminergic-hippocampal interactions in attentional boost (Shohamy \& Wagner, 2008; Wimmer et al., 2012), this hypothesis should be directly tested.

\section{Acknowledgement}

This study was supported by the National Development Agency (TÁMOP4.2.2.A-11/1/KONV-2012-0052).

\section{Abbreviations}

ABT, attentional boost test; ANT, attention network test; BIS-11, Barratt Impulsiveness Scale-11; HAM-D, Hamilton Depression Rating Scale; HSD, Honestly Significant Difference; L-DOPA, L-3,4-dihydroxyphenylalanine; LED, levodopa equivalent dose; MIDI, Minnesota Impulsive Disorders Interview; PD, Parkinson's disease; SOGS, South Oaks Gambling Screen; UPDRS, Unified Parkinson's Disease Rating Scale. 


\section{Conflict of interest}

The authors (S.K., H.N., E.L.G., O.K.) declare no conflict of interest.

\section{References}

Abler, B., Hahlbrock, R., Unrath, A., Grön, G. \& Kassubek, J. (2009) At-risk for pathological gambling: imaging neural reward processing under chronic dopamine agonists. Brain, 132, 2396-2402.

Allcock, L.M., Rowan, E.N., Steen, I.N., Wesnes, K., Kenny, R.A. \& Burn, D.J. (2009) Impaired attention predicts falling in Parkinson's disease. Parkinsonism Relat. D., 15, 110-115.

Antonini, A., Siri, C., Santangelo, G., Cilia, R., Poletti, M., Canesi, M., Caporali, A., Mancini, F., Pezzoli, G., Ceravolo, R., Bonuccelli, U. \& Barone, P. (2011) Impulsivity and compulsivity in drug-naive patients with Parkinson's disease. Movement Disord., 26, 464-468.

Baddeley, A., Eysenck, M.W. \& Anderson, M.C. (2009) Memory. Psychology Press, New York, NY, pp. 217-244.

Bennett, K.M., Waterman, C., Scarpa, M. \& Castiello, U. (1995) Covert visuospatial attentional mechanisms in Parkinson's disease. Brain, 118, 153-166.

Berridge, K.C. (2007) The debate over dopamine's role in reward: the case for incentive salience. Psychopharmacology, 191, 391-431.

Berridge, K.C. (2012) From prediction error to incentive salience: mesolimbic computation of reward motivation. Eur. J. Neurosci., 35, 1124-1143.

Brown, R.G. \& Marsden, C.D. (1988) Internal versus external cues and the control of attention in Parkinson's disease. Brain, 111, 323-345.

Canesi, M., Rusconi, M.L., Isaias, I.U. \& Pezzoli, G. (2012) Artistic productivity and creative thinking in Parkinson's disease. Eur. J. Neurol., 19, 468-472.

Christenson, G.A., Faber, R.J., de Zwaan, M., Raymond, N.C., Specker, S.M., Ekern, M.D., Mackenzie, T.B., Crosby, R.D., Crow, S.J. \& Eckert, E.D. (1994) Compulsive buying: descriptive characteristics and psychiatric comorbidity. J. Clin. Psychiat., 55, 5-11.

Cools, R. (2006) Dopaminergic modulation of cognitive function-implications for L-DOPA treatment in Parkinson's disease. Neurosci. Biobehav. $R ., 30,1-23$.

Cools, R. (2011) Dopaminergic control of the striatum for high-level cognition. Curr. Opin. Neurobiol., 21, 402-407.

Cools, R., Miyakawa, A., Sheridan, M. \& D'Esposito, M. (2010) Enhanced frontal function in Parkinson's disease. Brain, 133, 225-233.

Corbetta, M. \& Shulman, G.L. (2002) Control of goal-directed and stimulusdriven attention in the brain. Nat. Rev. Neurosci., 3, 201-215.

Cossa, F.M., Della Sala, S. \& Spinnler, H. (1989) Selective visual attention in Alzheimer's and Parkinson's patients: memory- and data-driven control. Neuropsychologia, 27, 887-892.

Cristinzio, C., Bononi, M., Piacentini, S., Albanese, A. \& Bartolomeo, P. (2012) Attentional networks in Parkinson's disease. Behav. Neurol., doi:10.3233/BEN-129020. [Epub ahead of print].

Dang, L.C., O’Neil, J.P. \& Jagust, W.J. (2012) Dopamine supports coupling of attention-related networks. J. Neurosci., 32, 9582-9587.

Della Sala, S., Di Lorenzo, G., Giordano, A. \& Spinnler, H. (1986) Is there a specific visuo spatial impairment in Parkinsonians? J. Neurol. Neurosur. Ps., 49, 1258-1265.

Driver, J. \& Baylis, G.C. (1989) Movement and visual attention: the spotlight metaphor breaks down. J. Exp. Psychol. Hum. Percept. Perform., 15, 448-456.

Fan, J., McCandliss, B.D., Sommer, T., Raz, A. \& Posner, M.I. (2002) Testing the efficiency and independence of attentional networks. J. Cognitive Neurosci., 14, 340-347.

Fan, J., McCandliss, B.D., Fossella, J., Flombaum, J.I. \& Posner, M.I. (2005) The activation of attentional networks. NeuroImage, 26, 471-479.

Fan, J., Gu, X., Guise, K.G., Liu, X., Fossella, J., Wang, H. \& Posner, M.I. (2009) Testing the behavioral interaction and integration of attentional networks. Brain Cognition, 70, 209-220.

First, M.B., Spitzer, R.L., Gibbon, M. \& Williams, J.B.W. (1996) Structured Clinical Interview for DSM-IV Axis I Disorders, Clinician Version (SCID$\mathrm{CV}$ ). American Psychiatric Press, Washington, DC.

Foerde, K. \& Shohamy, D. (2011) The role of the basal ganglia in learning and memory: insight from Parkinson's disease. Neurobiol. Learn. Mem., 96, 624-636.

Foerde, K., Braun, E.K. \& Shohamy, D. (2013) A trade-off between feedback-based learning and episodic memory for feedback events: evidence from Parkinson's disease. Neurodegener. Dis., 11, 93-101.
Folk, C.L., Remington, R.W. \& Johnston, J.C. (1992) Involuntary covert orienting is contingent on attentional control settings. J. Exp. Psychol. Hum. Percept. Perform., 18, 1030-1044.

Frank, M.J. (2005) Dynamic dopamine modulation in the basal ganglia: a neurocomputational account of cognitive deficits in medicated and nonmedicated Parkinsonism. J. Cognitive Neurosci., 17, 51-72.

Hahn, E., Ta, T.M., Hahn, C., Kuehl, L.K., Ruehl, C., Neuhaus, A.H. \& Dettling, M. (2011) Test-retest reliability of Attention Network Test measures in schizophrenia. Schizophr. Res., 133, 218-222.

Hamilton, M. (1960) A rating scale for depression. J. Neurol. Neurosur. Ps., 23, 56-62.

Hodgson, T.L., Dittrich, W.H., Henderson, L. \& Kennard, C. (1999) Eye movements and spatial working memory in Parkinson's disease. Neuropsychologia, 37, 927-938.

Hoehn, M.M. \& Yahr, M.D. (1967) Parkinsonism: onset, progression and mortality. Neurology, 17, 427-442.

Hollingshead, A.B. (1975) Four Factor Index of Social Status. Unpublished working paper, Department of Sociology, Yale University, New Haven, CT.

Housden, C.R., O’Sullivan, S.S., Joyce, E.M., Lees, A.J. \& Roiser, J.P. (2010) Intact reward learning but elevated delay discounting in Parkinson's disease patients with impulsive-compulsive spectrum behaviors. Neuropsychopharmacol., 35, 2155-2164.

Hughes, A.J., Daniel, S.E., Kilford, L. \& Lees, A.J. (1992) Accuracy of clinical diagnosis of idiopathic Parkinson's disease: a clinico-pathological study of 100 cases. J. Neurol. Neurosur. Ps., 55, 181-184.

Isaias, I.U., Siri, C., Cilia, R., De Gaspari, D., Pezzoli, G. \& Antonini, A. (2008) The relationship between impulsivity and impulse control disorders in Parkinson's disease. Movement Disord., 23, 411-415.

Kingstone, A., Klein, R., Morein-Zamir, S., Hunt, A., Fisk, J. \& Maxner, C. (2002) Orienting attention in aging and Parkinson's disease: distinguishing modes of control. J. Clin. Exp. Neuropsyc., 24, 951-967.

Koerts, J., Leenders, K.L. \& Brouwer, W.H. (2009) Cognitive dysfunction in non-demented Parkinson's disease patients: controlled and automatic behavior. Cortex, 45, 922-929.

Lang, A.E. \& Fahn, S. (1989) Assessment of Parkinson's disease. In Munsat, T.L. (Ed.), Quantification of Neurologic Deficit. Butterworth- Heinemann, Boston, Mass, pp. 285-309.

Lawrence, A.D., Evans, A.H. \& Lees, A.J. (2003) Compulsive use of dopamine replacement therapy in Parkinson's disease: reward systems gone awry? Lancet Neurol., 2, 595-604.

Leclercq, V. \& Seitz, A.R. (2012) Enhancement from targets and suppression from cues in fast task-irrelevant perceptual learning. Acta Psychol. (Amst.), 141, 31-38.

Lee, S.S., Wild, K., Hollnagel, C. \& Grafman, J. (1999) Selective visual attention in patients with frontal lobe lesions or Parkinson's disease. Neuropsychologia, 37, 595-604.

Lesieur, H.R. \& Blume, S.B. (1987) The South Oaks Gambling Screen (SOGS): a new instrument for the identification of pathological gamblers. Am. J. Psychiat., 144, 1184-1188.

Levy-Gigi, E. \& Kéri, S. (2012) Falling out of time: enhanced memory for scenes presented at behaviorally irrelevant points in time in Posttraumatic Stress Disorder (PTSD). PLoS ONE, 7, e42502.

Lin, J.Y., Pype, A.D., Murray, S.O. \& Boynton, G.M. (2010) Enhanced memory for scenes presented at behaviorally relevant points in time. PLoS Biol., 8, e1000337.

Marrocco, R.T. \& Davidson, M.C. (1998) Neurochemistry of attention. In Parasuraman, R. (Ed.), The Attentive Brain. MIT Press, Cambridge, MA.

Montague, P.R., Dayan, P. \& Sejnowski, T.J. (1996) A framework for mesencephalic dopamine systems based on predictive Hebbian learning. $J$. Neurosci., 16, 1936-1947.

Moustafa, A.A., Sherman, S.J. \& Frank, M.J. (2008) A dopaminergic basis for working memory, learning and attentional shifting in Parkinsonism. Neuropsychologia, 46, 3144-3156.

Müller, U., Suckling, J., Zelaya, F., Honey, G., Faessel, H., Williams, S.C., Routledge, C., Brown, J., Robbins, T.W. \& Bullmore, E.T. (2005) Plasma level-dependent effects of methylphenidate on task-related functional magnetic resonance imaging signal changes. Psychopharmacology, 180, 624633.

Muslimovic, D., Post, B., Speelman, J.D. \& Schmand, B. (2005) Cognitive profile of patients with newly diagnosed Parkinson disease. Neurology, 65, 1239-1245.

Muzzio, I.A., Kentros, C. \& Kandel, E. (2009) What is remembered? Role of attention on the encoding and retrieval of hippocampal representations. J. Physiol., 587, 2837-2854. 
Nagy, H., Levy-Gigi, E., Somlai, Z., Takáts, A., Bereczki, D. \& Kéri, S. (2012) The effect of dopamine agonists on adaptive and aberrant salience in Parkinson's disease. Neuropsychopharmacol., 37, 950-958.

Nieoullon, A. (2002) Dopamine and the regulation of cognition and attention. Prog. Neurobiol., 67, 53-83.

Patton, J.H., Stanford, M.S. \& Barratt, E.S. (1995) Factor structure of the Barratt impulsiveness scale. J. Clin. Psychol., 51, 768-774.

Posner, M.I. (2008) Measuring alertness. Ann. NY Acad. Sci., 1129, 193-199.

Rafal, R.D., Posner, M.I., Walker, J.A. \& Friedrich, F.J. (1984) Cognition and the basal ganglia. Separating mental and motor components of performance in Parkinson's disease. Brain, 107, 1083-1094.

Rao, H., Mamikonyan, E., Detre, J.A., Siderowf, A.D., Stern, M.B., Potenza, M.N. \& Weintraub, D. (2010) Decreased ventral striatal activity with impulse control disorders in Parkinson's disease. Movement Disord., 25, 1660-1669.

Riba, J., Krämer, U.M., Heldmann, M., Richter, S. \& Münte, T.F. (2008) Dopamine agonist increases risk taking but blunts reward-related brain activity. PLOS ONE, 3, e2479.

Robbins, T.W. (2002) The 5-choice serial reaction time task: behavioural pharmacology and functional neurochemistry. Psychopharmacology, 163, $362-380$.

Robbins, T.W. \& Everitt, B.J. (2007) A role for mesencephalic dopamine in activation: commentary on Berridge (2006). Psychopharmacology, 191, 433-437.

Samejima, K., Ueda, Y., Doya, K. \& Kimura, M. (2005) Representation of action-specific reward values in the striatum. Science, 310, 1337-1340.

Schultz, W., Dayan, P. \& Montague, P.R. (1997) A neural substrate of prediction and reward. Science, 275, 1593-1599.

Seitz, A.R. \& Watanabe, T. (2009) The phenomenon of task-irrelevant perceptual learning. Vision Res., 49, 2604-2610.

Seitz, A.R., Kim, D. \& Watanabe, T. (2009) Rewards evoke learning of unconsciously processed visual stimuli in adult humans. Neuron, 61, 700-707.

Shohamy, D. \& Wagner, A.D. (2008) Integrating memories in the human brain: hippocampal-midbrain encoding of overlapping events. Neuron, 60, 378-389.

Swallow, K.M. \& Jiang, Y.V. (2010) The Attentional Boost Effect: transient increases in attention to one task enhance performance in a second task. Cognition, 115, 118-132.
Swallow, K.M. \& Jiang, Y.V. (2011) The role of timing in the attentional boost effect. Atten. Percept. Psycho., 73, 389-404.

Swallow, K.M., Makovski, T. \& Jiang, Y.V. (2012) Selection of events in time enhances activity throughout early visual cortex. J. Neurophysiol., 108, 3239-3252.

Szamosi, A., Levy-Gigi, E., Kelemen, O. \& Kéri, S. (2013) The hippocampus plays a role in the recognition of visual scenes presented at behaviorally relevant points in time: evidence from amnestic mild cognitive impairment (aMCI) and healthy controls. Cortex, 49, 1892-1900.

Tomasi, D., Volkow, N.D., Wang, G.J., Wang, R., Telang, F., Caparelli, E.C., Wong, C., Jayne, M. \& Fowler, J.S. (2011) Methylphenidate enhances brain activation and deactivation responses to visual attention and working memory tasks in healthy controls. NeuroImage, 54, 31013110 .

Tomlinson, C.L., Stowe, R., Patel, S., Rick, C., Gray, R. \& Clarke, C.E. (2010) Systematic review of levodopa dose equivalency reporting in Parkinson's disease. Movement Disord., 25, 2649-2653.

Tosoni, A., Shulman, G.L., Pope, A.L., McAvoy, M.P. \& Corbetta, M. (2013) Distinct representations for shifts of spatial attention and changes of reward contingencies in the human brain. Cortex, 49, 17331749 .

Vandenbossche, J., Deroost, N., Soetens, E., Zeischka, P., Spildooren, J., Vercruysse, S., Nieuwboer, A. \& Kerckhofs, E. (2012) Conflict and freezing of gait in Parkinson's disease: support for a response control deficit. Neuroscience, 206, 144-154.

Voon, V., Gao, J., Brezing, C., Symmonds, M., Ekanayake, V., Fernandez, H., Dolan, R.J. \& Hallett, M. (2011) Dopamine agonists and risk: impulse control disorders in Parkinson's disease. Brain, 134, 1438-1446.

Wechsler, D. (1981) Wechsler Adult Intelligence Scale - Revised Manual. Psychological Corporation, New York, NY.

Weintraub, D. \& Nirenberg, M.J. (2013) Impulse control and related disorders in Parkinson's disease. Neurodegener. Dis., 11, 63-71.

Wimmer, G.E., Daw, N.D. \& Shohamy, D. (2012) Generalization of value in reinforcement learning by humans. Eur. J. Neurosci., 35, 1092-1104.

Yamada, T., Izyuuinn, M., Schulzer, M. \& Hirayama, K. (1990) Covert orienting attention in Parkinson's disease. J. Neurol. Neurosur. Ps., 53, $593-$ 596.

Zhou, S., Chen, X., Wang, C., Yin, C., Hu, P. \& Wang, K. (2012) Selective attention deficits in early and moderate stage Parkinson's disease. Neurosci. Lett., 509, 50-55. 